\title{
Fast spectroscopic mapping of two-dimensional quantum materials
}

\author{
Berk Zengin $\odot,{ }^{1}$ Jens Oppliger $\odot,{ }^{1}$ Danyang Liu $\odot,{ }^{1}$ Lorena Niggli $\odot,{ }^{1}$ Tohru Kurosawa $\odot,{ }^{2}$ and Fabian D. Natterer ${ }^{1, *}$ \\ ${ }^{1}$ Department of Physics, University of Zurich, Winterthurerstrasse 190, 8057 Zurich, Switzerland \\ ${ }^{2}$ Department of Physics, Hokkaido University, Sapporo 060-0810, Japan
}

(Received 25 March 2021; revised 19 June 2021; accepted 20 October 2021; published 10 November 2021)

\begin{abstract}
The discovery of quantum materials entails extensive spectroscopic studies that are carried out against multitudes of degrees of freedom, such as magnetic field, location, temperature, or doping. As this traditionally involves two or more serial measurement tasks, spectroscopic mapping can become excruciatingly slow. We demonstrate orders of magnitude faster measurements through our combination of sparse sampling and parallel spectroscopy. We exemplify our concept using quasiparticle interference imaging of $\mathrm{Au}(111)$ and $\mathrm{Bi}_{2} \mathrm{Sr}_{2} \mathrm{CaCu}_{2} \mathrm{O}_{8+\delta}$ (Bi2212), as two well-known model systems. Our method is accessible, straightforward to implement with existing setups, and can be easily extended to promote gate or field spectroscopy. In view of further substantial speed advantages, it is setting the stage to fundamentally promote the discovery of quantum materials.
\end{abstract}

DOI: 10.1103/PhysRevResearch.3.L042025

The late Freeman Dyson marveled at the luxury of our times in which scientific progress is strong both in idea and tool-driven research, one occasionally outdoing the other [1]. The field of quantum materials [2] is oddly related to these observations because it has been heavily pushed by theoretical advances to which their experimental counterpart is still playing catchup. This is partially owed to the rapid development of numerical methods that allow for an inspection of a vast parameter space in the hunt for materials but also due to the hard realities in their experimental discovery that deal with real constraints, such as degradation, sample size, environment, and the lack of proper characterization tools. The active feedback from experimental observations would positively guide the theoretical exploration of candidate materials, and it exposes the urgent need for suitable characterization tools to enable and keep up with future developments.

We examine the necessity for concepts in the field of spectroscopic mapping with the scanning tunneling microscope (STM) as a tool in the discovery of quantum materials. From the beginning of its conception, the STM has been both notorious for its slow measurement pace that is intrinsic to the concept and revered for continually evolving into the tool that provides essential insights into the workings of the microscopic world [3-6]. To expose an Achilles' heel of STM investigations, we exemplify here one prominent measurement mode that involves the combination of topography and spectroscopy. The two serial tasks of moving the tip from one location to the next and recording a spectrum by sweeping the

\footnotetext{
*Correspondence to: fabian.natterer@uzh.ch

Published by the American Physical Society under the terms of the Creative Commons Attribution 4.0 International license. Further distribution of this work must maintain attribution to the author(s) and the published article's title, journal citation, and DOI.
}

bias-voltage requires ample time. Related STM modes, such as gate mapping spectroscopy [6] or field-dependent spectroscopy [7], experience similar issues limiting measurement throughput that could be equally treated within the context laid out in this Rapid Communication. What unites these modes is their combination of at least two serial tasks that involve recording the spectroscopy for every increment of a free parameter, which can be topography, temperature, magnetic field, doping, strain, or any combination thereof. The enchaining of several serial tasks is what renders spectroscopic mapping excruciatingly slow and oftentimes imposes compromises that directly impact data quality or spectroscopic resolution.

The total mapping time depends on the product of time per spectrum and the number of recorded spectra, i.e., the number of increments of the free parameter. The trivial conclusion on how to exponentially speed up spectroscopic mapping could then be phrased as "measuring fewer spectra faster."

We exemplify the implementation of this concept using spectroscopic mapping in which a full spectrum would have traditionally been recorded at every topographic location. From a Fourier transform of those local density of states (LDOS) measurements, one also obtains quasiparticle interference (QPI) information [8-16] that provides insight into the band structure of quantum materials. Such QPI imaging requires the recording of a massive number of spectra that can occupy an STM for several days for a single QPI dataset [13]. Here, we combine our sparse sampling approach [17] with parallel spectroscopy [18] to exponentially speed spectroscopic mapping. While sparse sampling enables the recording of QPI maps using fundamentally fewer LDOS measurements, parallel spectroscopy works by increasing the speed of an individual spectrum.

Figure 1(a) illustrates our setup, which was reversibly added to an existing low-temperature STM (Createc) using a relay switch, a compensating capacitor, and a multifrequency 

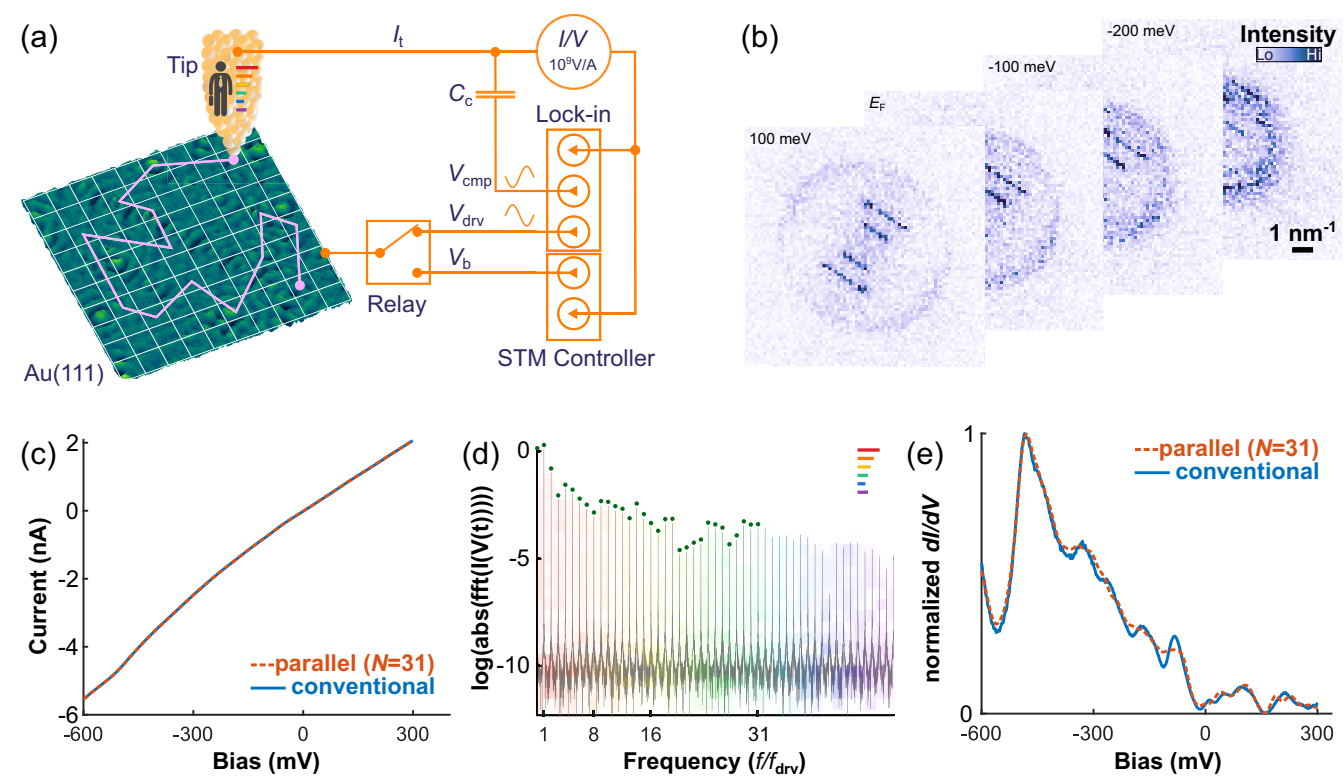

FIG. 1. Experimental setup and working principles used for fast spectroscopic mapping. (a) Wiring schematics of the components added to an existing scanning tunneling microscope (STM) setup, including a multifrequency lock-in amplifier, a relay switch, and a compensating capacitor. The grid and the purple line symbolize regular and traveling salesperson's (TSP's) paths that are both used for parallel spectroscopy and sparse sampling. The person inside the tip denotes the use of TSP and the colored stripes indicate the use of parallel spectroscopy. (b) Quasiparticle interference patterns obtained from Fourier transforms of local density of states (LDOS) maps, measured using parallel spectroscopy, showing the $\mathrm{Au}(111)$ surface state and indicating the sparsity in the quasiparticle interference (QPI) domain (setpoint: $V_{b}=$ $-200 \mathrm{mV}, V_{\mathrm{drv}}=600 \mathrm{mV}, f_{\mathrm{drv}}=250 \mathrm{~Hz}, I_{t}=800 \mathrm{pA}, t_{\mathrm{spc}}=100 \mathrm{~ms}$, and $\left.T=77 \mathrm{~K}\right)$. (c) Current-voltage characteristics $(I-V)$ of Au(111) measured with conventional spectroscopy (blue). The $I-V$ curve is characterized by one dominant and a few smaller nonlinearities that, upon application of a cosinusoidal drive, generate (d) higher-order harmonics, shown here by the Fourier transform of simulated $I[V(t)]$ traces. Their simultaneous demodulation (here, 31 dotted harmonics are used) enables the reconstruction of the original $I-V$ characteristics [orange dashed line in (c)] and (e) the conductance (orange dashes), shown here in comparison with the conventionally measured spectrum (blue).

lock-in amplifier (Intermodulation Products, MLA-3) [19]. The switch [20] enables to toggle between conventional STM operation and parallel spectroscopy, for which we require the compensating capacitor $\left(C_{\mathrm{c}}\right)$ to remove stray capacitancerelated displacement currents using $V_{\mathrm{cmp}}$ before reaching the preamplifier $[18,19]$. The sparse-sampling concept requires no additional hardware but only the control of the tip motion on a subset of randomized locations along a near optimal path (purple line) that was created in the spirit of a traveling salesperson [17]. Note that, for other spectroscopic mapping implementations, the locations would be substituted with temperature, magnetic field, doping, etc. (see example in the Supplemental Material [19]).

The amount of subsampling directly relates to the total mapping time and offers potential for further acceleration compared with conventional mapping when sparsity is high and sample symmetries are fully exploited [17]. STM investigations are amenable to compressive sensing concepts $[21,22]$ because the data structure is oftentimes highly sparse or compressible in one representation space. Here, this applies to the QPI pattern $[17,23]$, which consists of only a few nonzero values in the Fourier domain [Fig. 1(b)].

To speed up the measurement at each of those reduced number of locations, a cosinusoidal drive of amplitude $V_{\mathrm{drv}}$ and frequency $f_{\text {drv }}$ is applied onto the tunneling junction, which is characterized by nonlinearities in the current-voltage $(I-V)$ characteristic [Fig. 1(c)]. These nonlinearities lead to the generation of higher-order harmonics [Fig. 1(d)], whose parallel measurement (here, 31 amplitudes and phases) enables the reconstruction of their generating $I-V$ signature via an inverse Fourier transform [18]. Since the drive amplitude covers the energy range $\left(V_{b}-V_{d r v}\right.$ to $\left.V_{b}+V_{d r v}\right)$, this concept yields the full spectrum in a fraction of the time when compared with conventional spectroscopy that consisted in incrementally sweeping a voltage and recording the corresponding current/conductance. As QPI mapping requires LDOS measurements, which are proportional to the conductance, we also show a comparison of the conventional (blue) and a parallel measurement (orange dashed) of the latter [Fig. 1(e)], obtained for conceptualization by numerically applying $V_{\mathrm{drv}}$ onto the $I-V$ characteristics of panel (c). The $d I / d V$ spectrum described with 31 harmonics shows excellent agreement with the reference spectrum. More spectroscopic details may be achieved by using additional demodulators, by reducing the energy range that is covered by the drive voltage, or by strategically selecting harmonics with the strongest amplitudes to maximize the effect of the 31 demodulators [see nonmonotonic distribution of harmonics in Fig. 1(d)].

Figure 2 exemplifies how either of the two trivial speed promoters work independently for QPI imaging. In this example, we map the Shockley surface state of $\mathrm{Au}(111)$ by only using parallel spectroscopy (top row) or only sparse sampling (bottom row) on large grids. In the top row, we move the tip along a regular grid but measure each pixel using parallel spectroscopy. The reduced mapping time comes solely from the faster point spectra, taking only $\sim 100 \mathrm{~ms}$. 

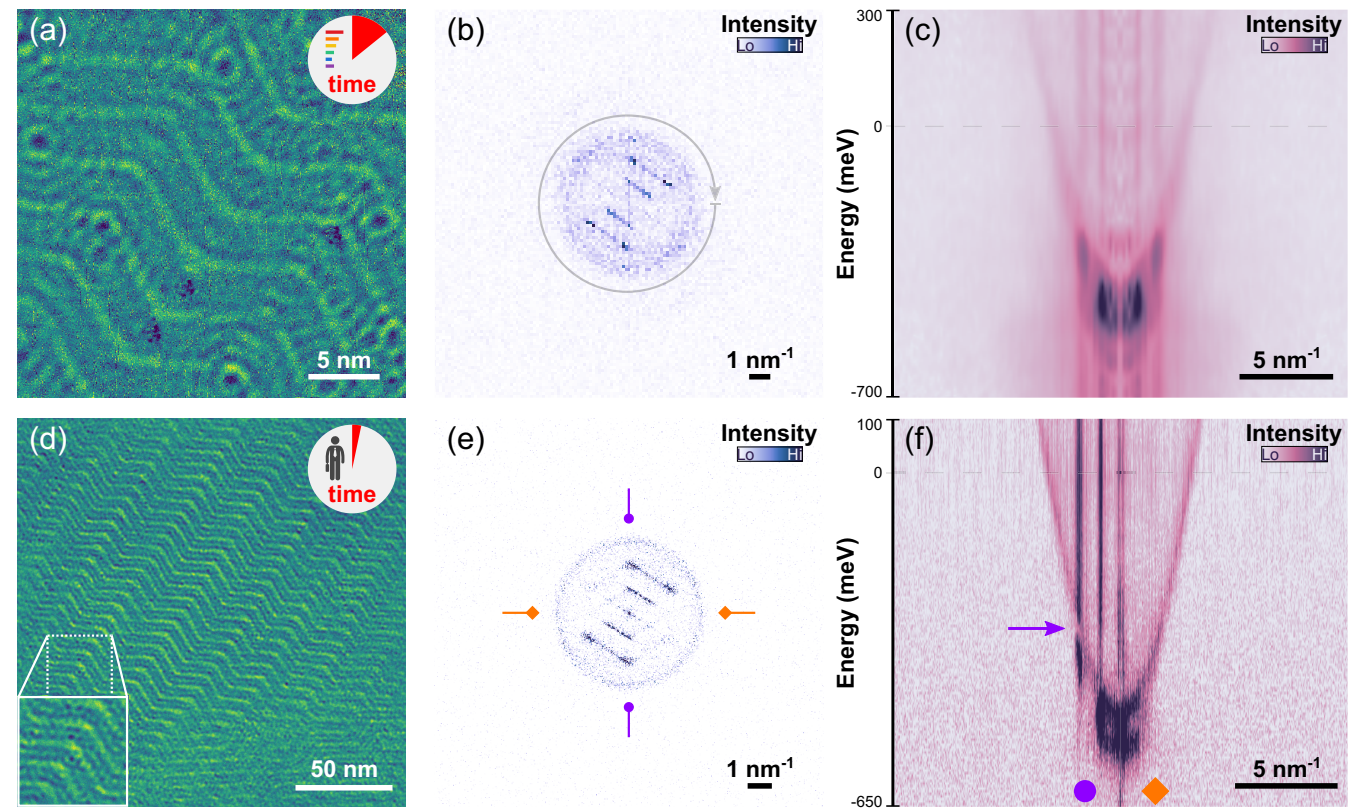

(e)

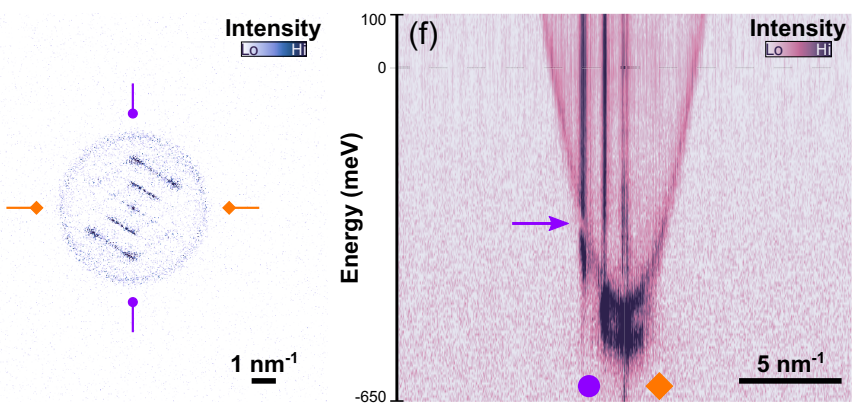

FIG. 2. Parallel spectroscopy (top row) and sparse sampling (bottom row) for quasiparticle interference (QPI) imaging, independently exemplified with $\mathrm{Au}(111)$. (a) Local density of states (LDOS) at $-100 \mathrm{mV}$, measured using parallel spectroscopy on a regular $512 \times 512$ grid, taking $10.3 \mathrm{~h}$ instead of $73 \mathrm{~h}$ (setpoint: $V_{b}=-200 \mathrm{mV}, V_{\mathrm{drv}}=600 \mathrm{mV}, f_{\mathrm{drv}}=250 \mathrm{~Hz}, I_{t}=800 \mathrm{pA}, t_{\mathrm{spc}}=100 \mathrm{~ms}$, and $T=70 \mathrm{~K}$ ). (b) QPI pattern obtained from a Fourier transform of (a). (c) Dispersion relation from azimuthally averaged QPI maps showing the nearly free electron band $\left(m^{*} / m_{e}=0.24 \pm 0.02\right)$ and the nondispersing herringbone reconstruction. (d) LDOS at $-100 \mathrm{mV}$, obtained from an inverse fast Fourier transform (FFT) of (e). (e) QPI pattern obtained from our sparse reconstruction using a $2 \%$ subsampled $2048 \times 2048$ grid of conventionally measured spectra, taking about $38 \mathrm{~h}$, instead of $48.5 \mathrm{~d}$ (setpoint: $V_{b}=-700 \mathrm{mV}, I_{t}=6 \mathrm{nA}, V_{\mathrm{mod}}=5 \mathrm{mV}, f_{\mathrm{mod}}=997 \mathrm{~Hz}, t_{\mathrm{spc}}=1.6 \mathrm{~s}$, and $T=4.2 \mathrm{~K}$ ). (f) Dispersion relation (5-point average) along the directions indicated in (e) $\left(\mathrm{m}^{*} / \mathrm{m}_{e}=0.221 \pm 0.002\right)$. Note the presence of a band gap when the surface state coincides with the herringbone periodicity - $312 \mathrm{mV}$ (arrow), reflecting the backfolding of the surface state due to a periodic potential.

This map was measured at $70 \mathrm{~K}$ to demonstrate compatibility in a large temperature range. On the bottom row, we use sparse sampling on a small selection of randomly chosen points but measure every spectrum using conventional point spectroscopy. Here, the speed-up comes from the reduced number of measurements. In both modes, we clearly see the standing wave patterns (a) and (d), showing the scattering of electron waves at discontinuities and which lead to the ring in the QPI maps (b) and (e). The energy resolved profiles (c) and (e) show the near-free electronlike behavior of the charge carriers in $\mathrm{Au}(111)$ and nondispersing features that are related to the $(22 \times \sqrt{3})$ herringbone reconstruction [24]. The latter acts as a periodic potential responsible for the opening of an energy gap $\sim-312 \mathrm{mV}$ $[25,26]$ [purple arrow in (e)]. Our measurements not only prove the independent viability of parallel spectroscopy and sparse sampling, but they can also reveal sufficient detail to access the physics of band folding that is difficult to spot with angle-resolved photoemission spectroscopy [27], and that has received considerable attention by the advent of twisted bilayer systems [28]. This further reinforces the singular role of QPI mapping for the high-resolution exploration of quantum materials.

In Fig. 3, we conclude our demonstration of fast spectroscopic mapping by combining parallel spectroscopy with sparse sampling. In addition to $\mathrm{Au}(111)$ (a)-(c), we demonstrate rapid QPI mapping with Bi2212 as one of the most notorious examples of the cuprate family [8,10-12,16,29-33].
Our combination of sparse sampling and parallel spectroscopy enables the compromise-free spectroscopic mapping of a large reciprocal space that we thoroughly map within minutes to only a few hours in what would have been previously a month-long measurement. The QPI maps reveal characteristic signatures of $\mathrm{Bi} 2212$, such as the Bragg peaks $\left(Q_{x}, Q_{y}\right)$, a supermodulation $\left(Q_{\mathrm{sm}}\right)$, and checkerboard structures $\left(q_{x}, q_{y}\right)$ (e). The dispersion plots (f) capture the dispersion relation along the supermodulation and Bragg peak directions and illustrate the size of our reciprocal space of $\sim 95 \mathrm{~nm}^{-1}$, achieved by our 2048 pixel grid spacing. The presence of Bragg peaks shows the excellent compatibility of our method with established data processing [12] and analysis approaches.

Additional speed advantage can be unlocked by further enhancing the sparse sampling and parallel spectroscopy contributions as the slightest improvement of either will have immediate impact on the total measurement time through their geometric relationship. For the sparse sampling case, the exploitation of sample symmetries could allow 100-fold fewer measurements [17]; while parallel spectroscopy becomes faster, the faster $f_{\mathrm{drv}}$ and the more demodulators are used [18].

Finally, our concepts can be easily extended to other spectroscopic modes in which the location would be replaced by charge-carrier concentration [19] or magnetic field. Therein, one would measure the spectroscopy at a random subset of that free parameter and utilize a suitable sparsifying basis for compressive sensing. Instead of the Fourier space, 

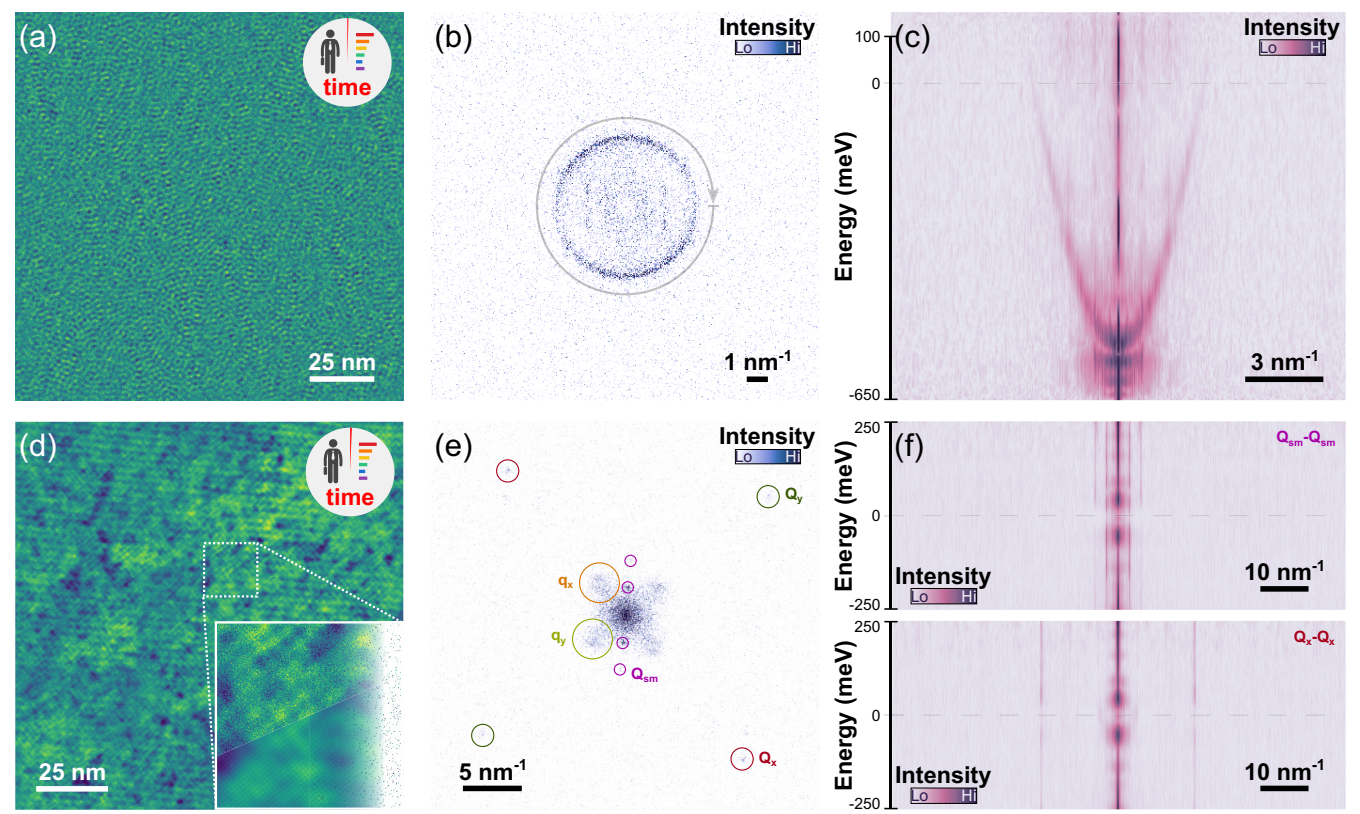

(e)
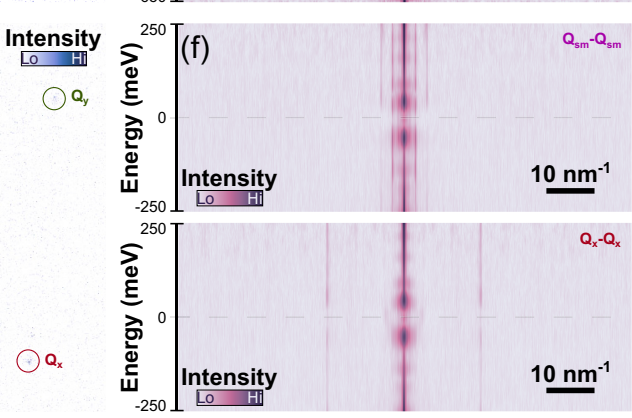

FIG. 3. Combination of parallel spectroscopy and sparse sampling. (a) Local density of states (LDOS) at $-100 \mathrm{mV}$, obtained from an inverse fast Fourier transform (FFT) of the quasiparticle interference (QPI) map in (b), which was (b) measured using the combined concept of parallel spectroscopy and sparse sampling on a $7 \%$ subsampled $1024 \times 1024$ grid on $\mathrm{Au}(111)$, taking $\sim 40$ min instead of $12 \mathrm{~d}$ (setpoint: $V_{b}=$ $-250 \mathrm{mV}, V_{\mathrm{drv}}=400 \mathrm{mV}, f_{\mathrm{drv}}=1600 \mathrm{~Hz}, I_{t}=800 \mathrm{pA}, t_{\mathrm{spc}}=20 \mathrm{~ms}$, and $T=4.3 \mathrm{~K}$ ). (c) Dispersion relation from azimuthally averaged QPI maps. (d) LDOS of Bi2212 at $0 \mathrm{mV}$, obtained from an inverse FFT of the QPI map in (e). The combination of parallel spectroscopy and sparse sampling on a $3 \%$ subsampled $2048 \times 2048$ grid took $6.5 \mathrm{~h}$ instead of $48.5 \mathrm{~d}$. The inset shows checkerboard, atomic contrast (enhanced by adding weight in QPI pattern), and the actual measurement locations (speckles on white gradient), also discernible by the Bragg peaks and scattering vectors in (e) and (f) (setpoint: $V_{b}=-100 \mathrm{mV}, V_{\mathrm{drv}}=400 \mathrm{mV}, f_{\mathrm{drv}}=250 \mathrm{~Hz}, I_{t}=400 \mathrm{pA}, t_{\mathrm{spc}}=100 \mathrm{~ms}$, and $T=4.2 \mathrm{~K}$ ). (e) Raw QPI pattern of Bi2212 at $0 \mathrm{mV}$, as obtained after sparse reconstruction of the parallel spectroscopy enabled measurement. The small circles denote the supermodulation $\left(Q_{\mathrm{sm}}\right)$, medium circles are the Bragg peaks $\left(Q_{x}, Q_{y}\right)$, and large circles are associated with checkerboard modulation $\left(q_{x}, q_{y}\right)$. (f) (top) Dispersion relation along the supermodulation $\left(Q_{\mathrm{sm}}\right)$ and (bottom) across the Bragg peaks $Q_{y}$ shown in (e). The time comparisons assume $1 \mathrm{~s}$ per spectrum for conventional mapping.

this could be a wavelet-based domain or a dictionary that properly encodes the intrinsic structure of the measured data, also making these mapping modes amendable to substantial speed improvements.

Since experimental discovery is ideally a process in which first impressions and trends of early measurements inform further steps in the exploration, our faster mapping concept will bring active control at the hands of the experimenter. This will not only provide a faster convergence to the understanding of materials but is also immediately practical to obtain proper statistics and coping with experimental challenges, such as tip-related instabilities, noisy lab settings, or the limited holdtime of one's cryostat.

All data are available in the main text or the Supplemental Material [19]. Full QPI maps are available upon reasonable request.
We acknowledge fruitful discussions with Riccardo Borgani, Daniel Forchheimer, Matthias Hengsberger, Kevin Hauser, and Johan Chang. We thank Qisi Wang for help with mounting the Bi2212 sample and Joe Stroscio for providing us with a gate map. F.D.N. thanks the Swiss National Science Foundation under Project No. PP00P2-176866 and ONR (N00014-20-1-2352) for generous support. D.L. thanks UZH Forschungskredit (FK-20-093), and T.K. acknowledges support by JSPS KAKENHI (Grant No. 19K03733).

F.D.N. conceived and supervised the work. B.Z. implemented parallel spectroscopy and J.O. sparse sampling. F.D.N., D.L., and B.Z. carried out the measurements. T.K. synthesized the Bi2212 crystal. D.L. and B.Z. prepared the samples. F.D.N., B.Z., J.O., and L.N. analyzed the data. F.D.N. wrote the manuscript.

Authors declare no competing interests.
[1] F. J. Dyson, Is science mostly driven by ideas or by tools? Science 338, 1426 (2012).

[2] F. Giustino, J. H. Lee, F. Trier, M. Bibes, S. M. Winter, R. Valentí, Y.-W. Son, L. Taillefer, C. Heil, A. I. Figueroa, B. Plaçais, Q. Wu, O. V. Yazyev, E. P. A. M. Bakkers, J. Nygård, P. Forn-Díaz, S. D. Franceschi, J. W. McIver, L. E. F. F. Torres, T. Low et al., The 2021 quantum materials roadmap, J. Phys. Mater. 3, 042006 (2021).
[3] G. Nunes and M. R. Freeman, Picosecond resolution in scanning tunneling microscopy, Science 262, 1029 (1993).

[4] X. H. Qiu, G. V. Nazin, and W. Ho, Vibrationally resolved fluorescence excited with submolecular precision, Science 299, 542 (2003).

[5] S. Baumann, W. Paul, T. Choi, C. P. Lutz, A. Ardavan, and A. J. Heinrich, Electron paramagnetic resonance of individual atoms on a surface, Science 350, 417 (2015). 
[6] Y. Zhao, J. Wyrick, F. D. Natterer, J. F. Rodriguez-Nieva, C. Lewandowski, K. Watanabe, T. Taniguchi, L. S. Levitov, N. B. Zhitenev, and J. A. Stroscio, Creating and probing electron whispering-gallery modes in graphene, Science 348, 672 (2015).

[7] F. Ghahari, D. Walkup, C. Gutiérrez, J. F. Rodriguez-Nieva, Y. Zhao, J. Wyrick, F. D. Natterer, W. G. Cullen, K. Watanabe, T. Taniguchi, L. S. Levitov, N. B. Zhitenev, and J. A. Stroscio, An on/off Berry phase switch in circular graphene resonators, Science 356, 845 (2017).

[8] E. W. Hudson, S. H. Pan, A. K. Gupta, K.-W. Ng, and J. C. Davis, Atomic-scale quasi-particle scattering resonances in $\mathrm{Bi}_{2} \mathrm{Sr}_{2} \mathrm{CaCu}_{2} \mathrm{O}_{8+\delta}$, Science 285, 88 (1999).

[9] L. Petersen, Ph. Hofmann, E. W. Plummer, and F. Besenbacher, Fourier transform-STM: Determining the surface fermi contour, J. Electron Spectrosc. Relat. Phenom. 109, 97 (2000).

[10] J. E. Hoffman, K. McElroy, D.-H. Lee, K. M. Lang, H. Eisaki, S. Uchida, and J. C. Davis, Imaging quasiparticle interference in $\mathrm{Bi}_{2} \mathrm{Sr}_{2} \mathrm{CaCu}_{2} \mathrm{O}_{8+\delta}$, Science 297, 1148 (2002).

[11] J. E. Hoffman, E. W. Hudson, K. M. Lang, V. Madhavan, H. Eisaki, S. Uchida, and J. C. Davis, A four unit cell periodic pattern of quasi-particle states surrounding vortex cores in $\mathrm{Bi}_{2} \mathrm{Sr}_{2} \mathrm{CaCu}_{2} \mathrm{O}_{8+\delta}$, Science 295, 466 (2002).

[12] M. J. Lawler, K. Fujita, J. Lee, A. R. Schmidt, Y. Kohsaka, C. K. Kim, H. Eisaki, S. Uchida, J. C. Davis, J. P. Sethna, and E.-A. Kim, Intra-unit-cell electronic nematicity of the high- $T_{\mathrm{c}}$ copperoxide pseudogap states, Nature (London) 466, 347 (2010).

[13] S. Grothe, S. Johnston, S. Chi, P. Dosanjh, S. A. Burke, and Y. Pennec, Quantifying Many-Body Effects by High-Resolution Fourier Transform Scanning Tunneling Spectroscopy, Phys. Rev. Lett. 111, 246804 (2013).

[14] H. Inoue, A. Gyenis, Z. Wang, J. Li, S. W. Oh, S. Jiang, N. $\mathrm{Ni}$, B. A. Bernevig, and A. Yazdani, Quasiparticle interference of the Fermi arcs and surface-bulk connectivity of a Weyl semimetal, Science 351, 1184 (2016).

[15] N. Morali, R. Batabyal, P. K. Nag, E. Liu, Q. Xu, Y. Sun, B. Yan, C. Felser, N. Avraham, and H. Beidenkopf, Fermi-arc diversity on surface terminations of the magnetic Weyl semimetal $\mathrm{Co}_{3} \mathrm{Sn}_{2} \mathrm{~S}$, Science 365, 1286 (2019).

[16] Y. Zhang, A. Mesaros, K. Fujita, S. D. Edkins, M. H. Hamidian, K. Ch'ng, H. Eisaki, S. Uchida, J. C. S. Davis, E. Khatami, and E.-A. Kim, Machine learning in electronic-quantummatter imaging experiments, Nature (London) 570, 484 (2019).

[17] J. Oppliger and F. D. Natterer, Sparse sampling for fast quasiparticle-interference mapping, Phys. Rev. Res. 2, 023117 (2020).

[18] R. Borgani, M. Gilzad Kohan, A. Vomiero, and D. B. Haviland, Fast Multifrequency Measurement of Nonlinear Conductance, Phys. Rev. Appl. 11, 044062 (2019).

[19] See Supplemental Material at http://link.aps.org/supplemental/ 10.1103/PhysRevResearch.3.L042025 for sample preparation, displacement current compensation, sparse-sampling matrices, additional spectroscopy information, and example of sparsely sampled gate map.

[20] F. D. Natterer, Waveform-sequencing for scanning tunneling microscopy based pump-probe spectroscopy and pulsed-ESR, MethodsX 6, 1279 (2019).

[21] E. J. Candès, J. K. Romberg, and T. Tao, Stable signal recovery from incomplete and inaccurate measurements, Commun. Pure Appl. Math. 59, 1207 (2006).

[22] D. L. Donoho, Compressed sensing, IEEE Trans. Inf. Theory. 52, 1289 (2006).

[23] Y. Nakanishi-Ohno, M. Haze, Y. Yoshida, K. Hukushima, Y. Hasegawa, and M. Okada, Compressed sensing in scanning tunneling microscopy/spectroscopy for observation of quasiparticle interference, J. Phys. Soc. Jpn. 85, 093702 (2016).

[24] Ch. Wöll, S. Chiang, R. J. Wilson, and P. H. Lippel, Determination of atom positions at stacking-fault dislocations on $\mathrm{Au}(111)$ by scanning tunneling microscopy, Phys. Rev. B 39, 7988 (1989).

[25] W. Chen, V. Madhavan, T. Jamneala, and M. F. Crommie, Scanning Tunneling Microscopy Observation of an Electronic Superlattice at the Surface of Clean Gold, Phys. Rev. Lett. 80, 1469 (1998).

[26] F. Reinert and G. Nicolay, Influence of the herringbone reconstruction on the surface electronic structure of $\mathrm{Au}(111)$, Appl. Phys. A 78, 817 (2004).

[27] J. Voit, L. Perfetti, F. Zwick, H. Berger, G. Margaritondo, G. Grüner, H. Höchst, and M. Grioni, Electronic structure of solids with competing periodic potentials, Science 290, 501 (2000).

[28] Y. Cao, V. Fatemi, S. Fang, K. Watanabe, T. Taniguchi, E. Kaxiras, and P. Jarillo-Herrero, Unconventional superconductivity in magic-angle graphene superlattices, Nature (London) 556, 43 (2018).

[29] Ch. Renner, B. Revaz, K. Kadowaki, I. Maggio-Aprile, and Ø. Fischer, Observation of the Low Temperature Pseudogap in the Vortex Cores of $\mathrm{Bi}_{2} \mathrm{Sr}_{2} \mathrm{CaCu}_{2} \mathrm{O}_{8+\delta}$, Phys. Rev. Lett. 80, 3606 (1998).

[30] S. H. Pan, E. W. Hudson, A. K. Gupta, K.-W. Ng, H. Eisaki, S. Uchida, and J. C. Davis, STM Studies of the Electronic Structure of Vortex Cores in $\mathrm{Bi}_{2} \mathrm{Sr}_{2} \mathrm{CaCu}_{2} \mathrm{O}_{8+\delta}$, Phys. Rev. Lett. 85, 1536 (2000).

[31] K. McElroy, R. W. Simmonds, J. E. Hoffman, D.-H. Lee, J. Orenstein, H. Eisaki, S. Uchida, and J. C. Davis, Relating atomic-scale electronic phenomena to wave-like quasiparticle states in superconducting $\mathrm{Bi}_{2} \mathrm{Sr}_{2} \mathrm{CaCu}_{2} \mathrm{O}_{8+\delta}$, Nature (London) 422, 592 (2003).

[32] H. Zhao, Z. Ren, B. Rachmilowitz, J. Schneeloch, R. Zhong, G. $\mathrm{Gu}, \mathrm{Z}$. Wang, and I. Zeljkovic, Charge-stripe crystal phase in an insulating cuprate, Nat. Mater. 18, 103 (2019).

[33] Q. Gu, S. Wan, Q. Tang, Z. Du, H. Yang, Q.-H. Wang, R. Zhong, J. Wen, G. D. Gu, and H.-H. Wen, Directly visualizing the sign change of $d$-wave superconducting gap in $\mathrm{Bi}_{2} \mathrm{Sr}_{2} \mathrm{CaCu}_{2} \mathrm{O}_{8+\delta}$ by phase-referenced quasiparticle interference, Nat. Commun. 10 , 1603 (2019). 